\title{
INVESTIGACIÓN EN EDUCACIÓN SUPERIOR: FACTOR CLAVE EN LA CONSTRUCCIÓN DEL CONOCIMIENTO.
}

\section{RESEARCH IN HIGHER EDUCATION: KEY FACTOR IN THE CONSTRUCTION OF KNOWLEDGE.}

\section{Wlamyr Palacios Alvarado ${ }^{1}$}

\section{Byron Medina Delgado²}

\section{Jorge Gómez Rojas ${ }^{3}$}

Ufps- Universidad del Magdalena

\section{RESUMEN}

La realidad social y económica de un país, región, localidad o de cualquier grupo social, es entendida a través de la investigación, a través de un proceso que estudia un fenómeno o problema mediante la aplicación de los enfoques cuantitativo, cualitativo o mixto, que están encaminados hacia la generación de conocimiento y en donde las instituciones de educación superior desempeñan un rol preponderante, reflexión que originó el tema del presente artículo. El objetivo es resaltar la investigación en la educación superior como factor clave en la construcción y generación de $1 \quad$ Vinculación: Universidad Francisco de Paula Santander, Cúcuta, Colombia. Cargo: Docente - Investigador Orcid: https://orcid.org/0000-0002-4292-4178 Email: wlamyrpalacios@ufps.edu.co

2 Vinculación: Universidad Francisco de Paula Santander, Cúcuta, Colombia.Cargo: Docente - Investigador Orcid: https://orcid.org/0000-0003-0754-8629 Email: byronmedina@ufps.edu.co

3 Vinculación: Universidad del Magdalena

Santa Marta, Colombia. Cargo: Docente - Investigador

Orcid: https://orcid.org/0000-0002-0840-8743

Email:jgomez@unimagdalena.edu.co conocimiento. La metodología es cualitativa y cuantitativa, aplica investigación exploratoria basada en fuentes secundarias de información relacionadas con el tema objeto de estudio. Los resultados señalan que países como Argentina, México, Chile y Venezuela han incrementado el número de investigadores entre el 2010 y el 2013 y, Colombia y Uruguay han disminuido el total de investigadores en ese período; además, aparece una baja actitud hacia la investigación por parte de instituciones y estudiantes de educación superior. Finalmente, se concluye que las instituciones de educación superior están obligadas a desarrollar planes, programas y estructuras curriculares centradas en la investigación, con el propósito de que estudiantes desarrollen competencias científicas para aprender a aprender, construir conocimiento y resolver los problemas del entorno socio-económico. 


\section{PALABRAS CLAVE}

Fenómeno, necesidades, oportunidades, proceso, actitud.

\section{ABSTRACT}

The social and economic reality of a country, region, locality or of any social group, is understood through research, through a process that studies a phenomenon or problem through the application of quantitative, qualitative or mixed approaches, which They are directed towards the generation of knowledge and where higher education institutions play a preponderant role, a reflection that originated the subject of this article. The objective is to highlight research in higher education as a key factor in the construction and generation of knowledge. The methodology is qualitative and quantitative, it applies exploratory research based on secondary sources of information related to the subject under study. The results indicate that countries such as Argentina, Mexico, Chile and Venezuela have increased the number of researchers between 2010 and 2013 and Colombia and Uruguay have decreased the total number of researchers in that period; In addition, there is a low attitude towards research on the part of higher education institutions and students. Finally, it is concluded that higher education institutions are obliged to develop curricular plans, programs and structures focused on research, with the purpose that students develop scientific competencies to learn to learn, build knowledge and solve problems in the socioeconomic environment.

\section{KEY WORDS}

Phenomenon, needs, opportunities, process, attitude.

\section{INTRODUCCIÓN}

En el proceso de conocimiento está implicada la rigurosidad y legitimidad social con fundamento en la metodología de investigación dentro de las perspectivas cualitativa, cuantitativa o mixta, con el propósito de construir conocimiento a partir del análisis sistemático, riguroso y formal de parte de las Instituciones de Educación Superior (IES), públicas o privadas, en donde prevalecen teorías y metodologías para comprender la realidad contextual.

En este sentido, aparece la investigación educativa cuyo propósito es conocer e interpretar detalladamente y a profundidad un problema de conocimiento, ya sea una necesidad insatisfecha o una oportunidad desaprovechada, brindar soluciones y difundir los descubrimientos hallados durante la investigación, precisiones que han originado el presente artículo cuyo objetivo es resaltar la investigación en la educación superior como factor clave en la construcción y generación de conocimiento.

El texto intenta sintetizar y recopilar teorías de la investigación, explicar la perspectiva cualitativa, cuantitativa o mixta y aportar algunas sugerencias en el diseño metodológico de la investigación, de acuerdo con el problema y los objetivos de la investigación.

En otra sección, se analiza la investigación en la educación superior dentro del marco de los protocolos que permitan construir e intercambiar conocimiento en las instituciones de educación superior, lo cual está previsto por el Sistema Nacional de Acreditación SNA, (2014) para la acreditación de programas en educación.

Esencialmente, el estudio aplica la metodología basada en las perspectivas cualitativa $y$ cuantitativa, mediante investigación tipo exploratoria, a través de la búsqueda de fuentes secundarias de información que permitan mostrar resultados en cuanto a la productividad científica y actitudes hacia la investigación. 


\section{MARCO TEÓRICO}

\subsection{Teorías sobre investigación}

Los fenómenos, sucesos, situaciones y/o ambientes generan ideas que son objeto de investigación y constituyen una primera aproximación a la realidad, contexto en el que pueden estar involucradas necesidades insatisfechas u oportunidades sin aprovechar, lo que en otras palabras representan el problema de la investigación.

Esencialmente, la investigación conforme lo señalan Hernández, et. al. (2014), es un "conjunto de procesos sistemáticos, críticos y empíricos que se aplican al estudio de un fenómeno o problema" (p.04), procesos que aplican el enfoque cuantitativo y el enfoque cualitativo o mixto y, que están encaminados hacia la generación de conocimiento.

Por su parte, Corona (2016) señala que la investigación es un proceso metodológico en el cual se indaga e investiga de forma exhaustiva sobre un problema de interés para el investigador, ya sea bajo el enfoque cualitativo, cuantitativo o socio-crítico, con fines encaminados a la resolución de dicho problema en el ámbito científico, humanístico y tecnológico.

Estosenfoquestienensus propias características, el enfoque cuantitativo de investigación se remite a conteos numéricos y métodos matemáticos, es secuencial y probatorio, según Hernández et al. (2014), tiene un proceso lógico y cronológico, cada etapa precede a la siguiente, el orden es riguroso, se inicia con una idea que se delimita para posterior formulación de objetivos y preguntas de investigación, se revisa la literatura y se construye un marco teórico, de acuerdo con la fuente citada.

De este modo, las preguntas permiten establecer hipótesis, determinar variables, diseñar un plan para probarlas, medir las variables en un determinado contexto, analizar las mediciones obtenidas utilizando métodos estadísticos y puntualizar conclusiones.

Debe señalarse que el enfoque cualitativo de investigación posee sus propias características, hace referencia a la naturaleza, carácter y propiedades de los fenómenos según Niglas (como se citó en Hernández et al. 2014), los estudios cualitativos pueden desarrollar preguntas e hipótesis antes, durante o después de la recolección y el análisis de los datos, con frecuencia, estas actividades sirven, primero, para descubrir cuáles son las preguntas de investigación más importantes, y después, para perfeccionarlas y responderlas, según Hernández et al. (2014).

Este enfoque cualitativo también se conoce como investigación naturalista, fenomenológica, interpretativa o etnográfica, este enfoque utiliza la recolección y análisis de los datos para afinar las preguntas de investigación o revelar nuevas interrogantes en el proceso de interpretación; es así que estos enfoques cuantitativo y cualitativo tienen claras diferencias, el enfoque cualitativo busca principalmente la "dispersión o expansión" de los datos e información, mientras que el enfoque cuantitativo pretende "acotar" intencionalmente la información, medir con precisión las variables del estudio, de acuerdo con Hernández et al. (2014).

Contextualizado el problema a investigar, se debe establecer cuál es el "estado del arte" del problema que se quiere investigar, en este sentido, para cada investigador el mismo problema tiene varios "niveles" de conocimiento de acuerdo con la experiencia y vivencias; según Medina (2007), este proceso implica hacer una observación; la percepción del problema a investigar depende de la postura filosófica y epistemológica que el investigador tenga, paradigma cualitativo o cuantitativo, de esta manera fundamenta sus marcos teóricos apoyado en modelos, leyes y campos del conocimiento, según la fuente citada. 


\subsection{La investigación en la educación superior}

La investigación educativa y pedagógica se manifiesta de dos maneras, en primer lugar, en los temas que se investigan, los maestros enfatizan por aspectos más vinculados a la práctica pedagógica y a las disciplinas escolares; en segundo lugar, las comunidades científicas se encuentran en la frontera del conocimiento preocupadas por aportar con solidez teórica y metodológica al desarrollo de las ciencias de la educación, de acuerdo con Calvo, Camargo y Pineda (2008).

De acuerdo con lo anterior, no significa que los maestros no produzcan conocimiento, significa que esta producción es de distinto tipo según los temas y formas de abordaje, lo que implica redes de información abiertas, para permitir un acercamiento entre los dos tipos de prácticas, la pedagógica y la científica, conforme con la fuente citada; por otra parte, los maestros a su vez enfatizan en la sistematización y recuperación de experiencias y así orientar la reflexión por el camino del cambio y la innovación, en donde los protocolos no se corresponden con lo que hacen los investigadores, según Calvo, Camargo y Pineda (2008).

Se requiere de protocolos que permitan intercambiar conocimiento con la producción de quienes realizan investigación, como actividad profesional a través de la construcción del conocimiento en las IES, lo cual está previsto por el Sistema Nacional de Acreditación SNA, (2014) para la acreditación de programas en educación, proceso de alta calidad que se refiere fundamentalmente a cómo una institución y sus programas orientan su deber ser hacia un ideal de excelencia, y pueden mostrar alta calidad mediante resultados específicos, tradición consolidada, impacto y reconocimiento social, según SNA (2014).
Por tal motivo, las características de alta calidad de una IES, desde la perspectiva de la acreditación, orientan su acción hacia la investigación científica, tecnológica, humanística y artística, en sintonía con el saber universal y con alta visibilidad y hacia los procesos formativos flexibles e interdisciplinarios para el desarrollo de conocimientos, capacidades y habilidades requeridos en las actuales dinámicas sociales y laborales.

Las precisiones anteriores están previstas en la Ley 30 de 1992, que organiza el servicio público de la educación superior, la cual señala en su Artículo 18 "Son instituciones universitarias 0 escuelas tecnológicas, aquellas facultadas para adelantar programas de formación en ocupaciones, programas de formación académica en profesiones o disciplinas y programas de especialización. Son universidades las reconocidas actualmente como tales y las instituciones que acrediten su desempeño con criterio de universalidad en las siguientes actividades: la investigación científica o tecnológica; la formación académica en profesiones o disciplinas; y la producción, desarrollo y transmisión del conocimiento y de la cultura universal y nacional".

La reflexión anterior, resalta la investigación para la construcción del conocimiento, factor característico en la llamada sociedad del conocimiento que genera ventajas competitivas a partir del conocimiento, el capital intelectual y de los activos intangibles, escenario en el que la educación superior desempeña un papel considerable en el desarrollo del capital intelectual, a través de procesos de enseñanza aprendizaje en la nueva sociedad, definida por Crespo, citada en Rengifo-Millán 2015, como "el paso de una sociedad basada en la producción de bienes materiales, a una sociedad de la era de la información en la que predomina la adquisición, el intercambio y la producción de 
nuevos conocimientos" (Rengifo-Millán, 2015, p.811).

\section{METODOLOGÍA}

El propósito del presente artículo es resaltar la importancia de la investigación en la educación superior como un factor clave para la construcción del conocimiento; su desarrollo se basa en un criterio cualitativo, considerando que "los planteamientos cualitativos están enfocados en profundizar en los fenómenos explorándolos desde la perspectiva de los participantes" (Hernández, Fernández y Baptista, 2014, p.376).

Inherente al criterio cualitativo, el análisis aborda la etnografía, de acuerdo con Rusque (2003), referente a la cultura hacia la investigación en las IES; en este orden de ideas, con base en fuentes secundarias de información relacionadas con el tema de la investigación, el proceso metodológico aplica una investigación exploratoria según fuentes secundarias de información sobre temas relacionados con investigación, lo que corresponde a un primer nivel de conocimiento con el propósito de "aumentar familiaridad del investigador con el fenómeno que va a investigar, aclarar conceptos, establecer preferencias para posteriores investigaciones" (Méndez, 2007, p.229).

\section{RESULTADOS}

Desde el 2014 hasta la fecha más de 30 países incrementaron su inversión en investigación, sin embargo, todavía hay muchos que siguen siendo dependientes de las tecnologías y conocimientos técnicos procedentes del extranjero, de acuerdo con UNESCO (2021 a), es por ello que los países requieren invertir más en investigación e innovación, además, algunos países están mejorando también la condición socioeconómica de los investigadores con la adopción de medidas de aumento de salarios y de otro tipo, según la fuente citada; así mismo, desde el 2014 han aumentado considerablemente en el mundo el número de investigadores y el índice de la colaboración científica internacional, según UNESCO (2021 a).

Se suma a lo anterior, que son numerosos los países de América Latina que cuentan con instrumentos de políticas en materia de ciencia, tecnología e innovación, según el informe de la UNESCO sobre la ciencia, se experimentó un fuerte aumento en la participación de América Latina en la enseñanza superior, así como en la disponibilidad de recursos humanos en el ámbito de la investigación y desarrollo (I+D) y de la producción científica, de acuerdo con UNESCO (2021 b); además, los países de la región no tratan en general de ser competitivos a nivel internacional en el campo de las actividades de alta tecnología, según la fuente citada.

En este orden de ideas, de acuerdo con UNESCO (2021 b), se observa que países como Argentina, México, Chile y Venezuela han incrementado el número de investigadores en el cuatrienio del 2010 al 2013 y, Colombia y Uruguay han disminuido el total de investigadores en ese período, como muestra la Tabla 1. 
Tabla 1. Número de investigadores por país en América Latina 2010-2013.

\begin{tabular}{|c|c|c|}
\hline País & $\mathbf{2 0 1 0}$ & $\mathbf{2 0 1 3}$ \\
\hline Brasil & 138.653 & --- \\
\hline Argentina & 45.200 & 51.685 \\
\hline México & 42.000 & 43.592 \\
\hline Colombia & 8.500 & 7.702 \\
\hline Chile & 6.000 & 6.803 \\
\hline Venezuela & 6.000 & 8.686 \\
\hline Uruguay & 2.000 & 1.803 \\
\hline
\end{tabular}

Fuente: Instituto de estadística de la UNESCO 2021.

La Figura 1 presenta el número de investigadores por cada mil miembros de la población activa en 2012, la cual compara algunos países de América Latina con los que tienen mayor número de investigadores a nivel mundial.

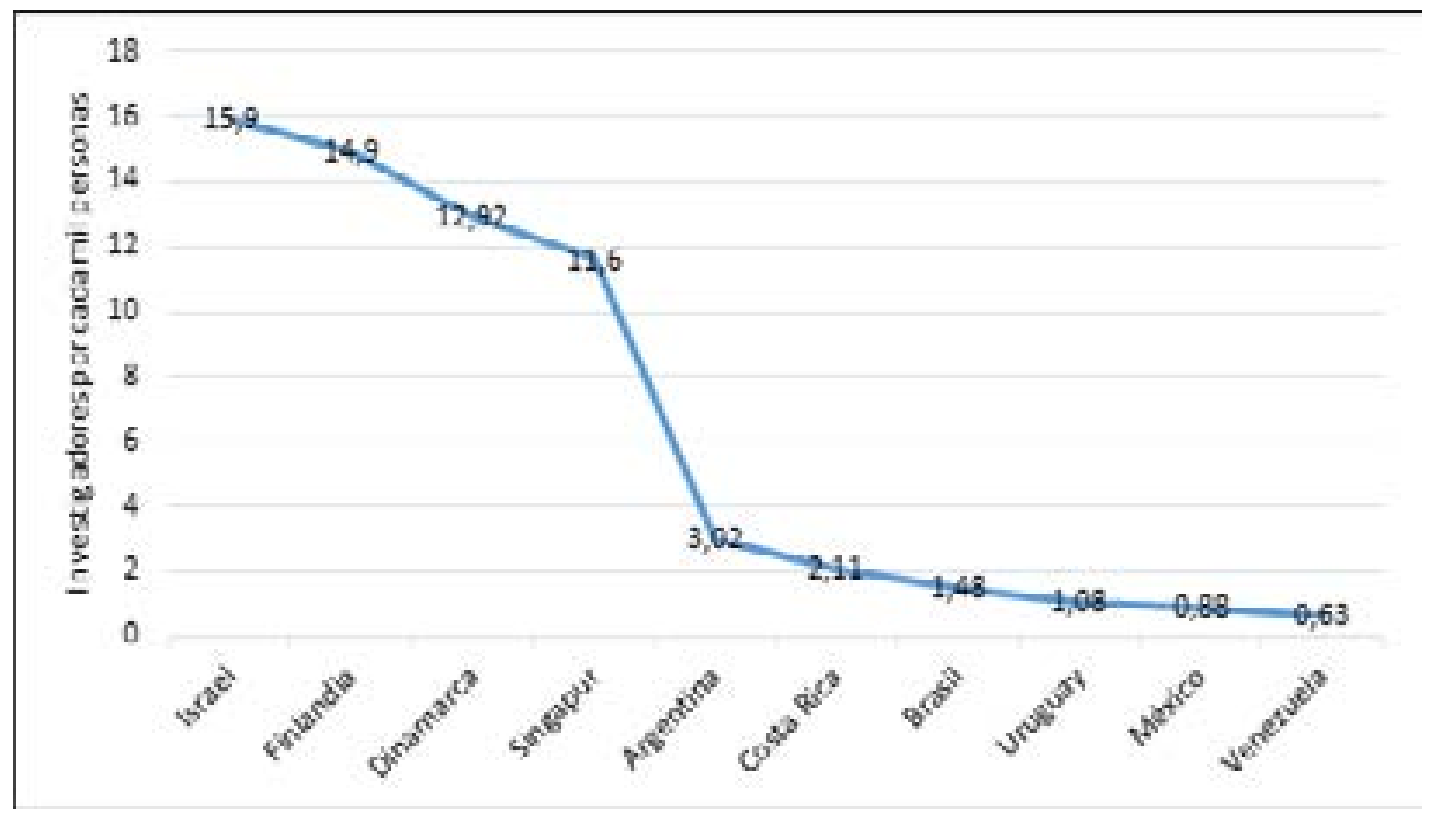

Figura 1. Comparativo de investigadores de la población activa 2012.

Fuente: UNESCO (2021 b)

Los resultados de la Figura 1, evidencian considerables diferencias entre los países con mayor número de investigadores por cada mil miembros de población activa en 2012, al comparar contra los resultados de América Latina en esta materia, escenarios en donde las IES poseen una posición de liderazgo cuando están contextualizadas en espacios sociales, con características temporales definidas por circunstancias culturales que les imprimen un carácter distintivo, convirtiéndose así singularmente en una diversidad dentro de la generalidad macro-social que las contiene, conforme lo señala Colina (2007). 
A nivel nacional, se realiza en 2014 la investigación a una población de 52.724 estudiantes, de segundo a décimo semestre de siete universidades, con el propósito de establecer la actitud hacia la investigación, mediante muestreo aleatorio simple, obteniendo una muestra final de 352 estudiantes, ponderada de acuerdo con el peso relativo del número de estudiantes en cada institución, conforme lo señalan Rojas y Méndez (2017).

Se aplicaron los instrumentos en dos universidades de carácter oficial: Universidad
Industrial de Santander y las Unidades Tecnológicas de Santander y, cinco universidades de carácter no oficial: Universidad Autónoma de Bucaramanga, Universidad Santo Tomás, Universidad Pontificia Bolivariana, Universitaria de Investigación y Desarrollo y, Universidad Cooperativa de Colombia, siendo las universidades más representativas del área metropolitana de Bucaramanga, Colombia y cuyos resultados que aparecen en Rojas y Méndez (2017) se presentan a continuación.

Figura 2. Índice general de actitud hacia la investigación.

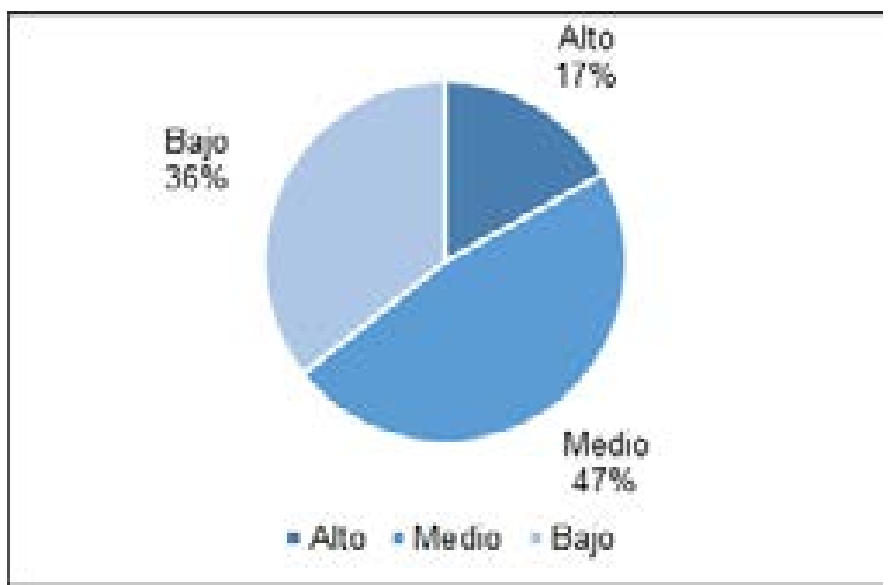

Fuente: Rojas y Méndez (2017).

En general, se obtuvo un alto grado de actitud en el $17,1 \%$ del total de estudiantes de pregrado, medio en el $47,4 \%$ y bajo en el $35,5 \%$ restante.
Los datos muestran una brecha considerable entre los estudiantes de actitud baja y alta, de acuerdo con Rojas y Méndez (2017).

Figura 3. Incidencia de institución en las actitudes hacia la investigación.

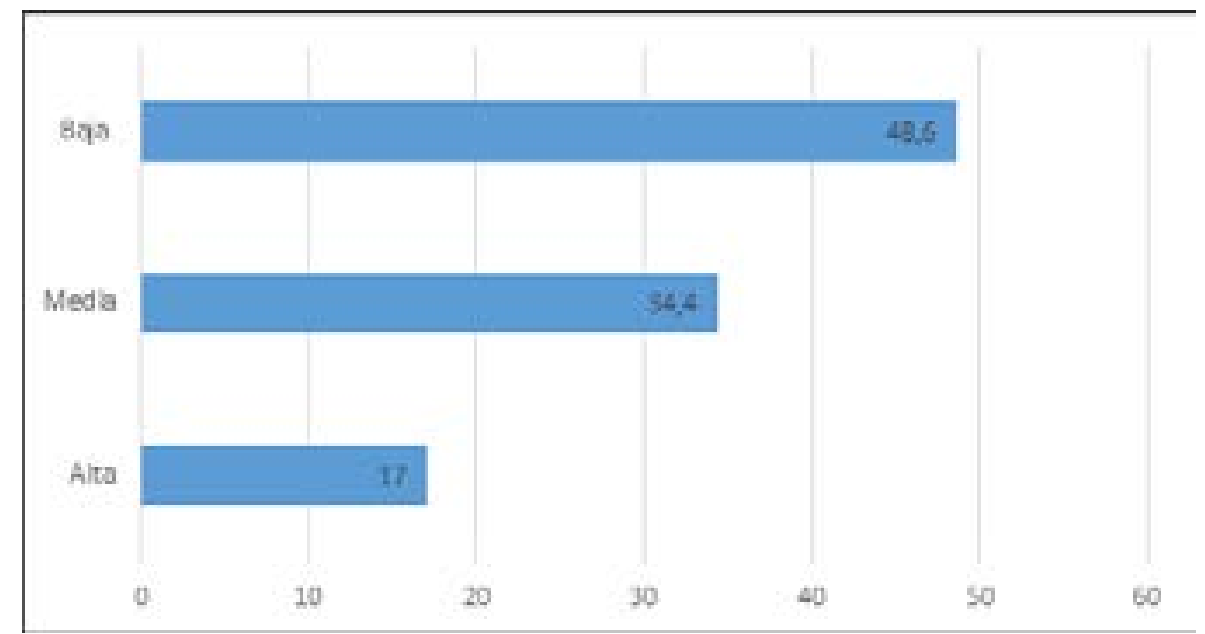

Fuente: Rojas y Méndez (2017). 
Al sumar las variables en subíndice de incidencia institucional, es el grupo de variables que menos aporta a la buena actitud, de acuerdo con la fuente citada. A pesar de la dispersión de las respuestas individuales, en el $48,6 \%$ del total de estudiantes se puntúa con baja incidencia, mientras que solo el 17,0 \% reporta alta incidencia sobre los aspectos institucionales y el $34,4 \%$ media incidencia.

\section{CONCLUSIONES}

Las instituciones de educación superior deben desarrollar planes, programas y estructuras curriculares centradas en la investigación, con el propósito de que los estudiantes desarrollen competencias científicas para aprender a aprender, construir conocimiento y resolver los problemas el entorno socio-económico.

Con base en la actitud de los estudiantes hacia la formación en investigación se evidencia que las universidades, siendo los mayores centros de producción científica en el país, no logran consolidar en el estudiante una cultura de la investigación.

Según la interpretación del contexto, enfoque, problema y objetivos de estudio, por parte del investigador, se aplica la perspectiva cualitativa, cuantitativa o mixta, considerando que, desde las técnicas y los métodos de análisis, la complementariedad de los métodos no solo es posible, sino enriquecedora para mejorar la capacidad investigadora.

El desarrollo de la ciencia en el país depende del grado de actitud hacia la investigación, por parte de las instituciones de educación superior y de sus estudiantes.

La formación en investigación es pertinente con el desarrollo humano y su aplicabilidad conduce a la construcción del conocimiento en la sociedad.

\section{REFERENCIAS BIBLIOGRÁFICAS}

Calvo, Gloria; Camargo Abello, Marina; Pineda Báez, Clelia, (2008), ¿Investigación educativa o investigación pedagógica? El caso de la investigación en el Distrito Capital Magis. Revista Internacional de Investigación en Educación, vol. 1, núm. 1, pp. 163-173 Pontificia Universidad Javeriana Bogotá, Colombia.

Colina, Lesbia, (2007), La investigación en la educación superior y su aplicabilidad social. Laurus, vol. 13 , núm. 25 , pp. 330353 Universidad Pedagógica Experimental Libertador Caracas, Venezuela.

Corona Lisboa, José, (2016), Investigación científica. A manera de reflexión, MediSur, vol. 14, núm. 3, pp. 1-2 Universidad de Ciencias Médicas de Cienfuegos Cienfuegos, Cuba.

Hernández Sampieri, R., Fernández Collado, C., Baptista Lucio, M., (2014), Metodología de la Investigación, Sexta Edición, Mcgraw-Hill / Interamericana Editores, S.A., México.

Medina Carrillo, L., (2007), Como plantear un problema de investigación y seleccionar un diseño de estudio apropiado, Archivos en Medicina Familiar, vol. 9, núm. 3, pp. 127-132 Asociación Latinoamericana de Profesores de Medicina Familiar A.C. México, Organismo Internacional.

Méndez, Carlos, (2007), Metodología, Diseño y desarrollo del proceso de investigación con énfasis en ciencias empresariales, $4^{\circ}$ edición, Editorial Limusa, Bogotá D.C.

Organización de las Naciones Unidas para la Educación, la Ciencia y la Cultura, UNESCO, (2021 a), Más colaboración científica y un gasto en investigación desigual. 
Organización de las Naciones Unidas para la Educación, la Ciencia y la Cultura, UNESCO, (2021 b), Panorámica regional: América Latina y el Caribe, Informe de la UNESCO sobre la Ciencia hacia 2030.

Rengifo-Millán, M. (2015). La globalización de la sociedad del conocimiento y la transformación universitaria. Revista Latinoamericana de Ciencias Sociales, Niñez y Juventud, 13 (2), pp. 809-822.

Rojas Betancur, Mauricio; Méndez Villamizar, Raquel, (2017), Procesos de formación en investigación en la universidad: ¿Qué le queda a los estudiantes? Sophia, vol. 13, núm. 2, pp. 53-69 Universidad La Gran Colombia Quindío, Colombia.

Rusque, Ana María, (2003), De la diversidad a la unidad en la investigación cualitativa, Primera Edición, Vadel Editores, Valencia, España.

Sistema Nacional de Acreditación SNA, (2014), Lineamientos para la acreditación institucional, Consejo Nacional de Acreditación Bogotá, D.C., Colombia. 